\title{
Assessment of somatic single-nucleotide variation in brain tissue of cases with schizophrenia
}

\author{
John F. Fullard ${ }^{1,2}$, Alexander W. Charney ${ }^{1,2}$, Georgios Voloudakis ${ }^{1}$, Andrew V. Uzilov $\mathbb{1}^{2,3}$, Vahram Haroutunian $\mathbb{1}^{1,4}$ and \\ Panos Roussos (1,2,4
}

\begin{abstract}
The genetic architecture of schizophrenia (SCZ) includes numerous risk loci across a range of frequencies and sizes, including common and rare single-nucleotide variants and insertions/deletions (indels), as well as rare copy number variants (CNVs). Despite the clear heritability of the disease, monozygotic twins are discordant for SCZ at a significant rate. Somatic variants - genetic changes that arise after fertilization rather than through germline inheritance-are widespread in the human brain and known to contribute to risk for both rare and common neuropsychiatric conditions. The contribution of somatic variants in the brain to risk of SCZ remains to be determined. In this study, we surveyed somatic single-nucleotide variants (SSNVs) in the brains of controls and individuals with SCZ ( $n=10$ and $n=$ 9, respectively). From each individual, whole-exome sequencing (WES) was performed on DNA from neuronal and non-neuronal nuclei isolated by fluorescence activated nuclear sorting (FANS) from frozen postmortem prefrontal cortex (PFC) samples, as well as DNA extracted from temporal muscle as a reference. We identified an increased burden of sSNVs in cases compared to controls (SCZ rate $=2.78$, control rate $=0.70 ; P=0.0092$, linear mixed effects model), that included a higher rate of non-synonymous and loss-of-function variants (SCZ rate $=1.33$, control rate $=$ $0.50 ; P=0.047$, linear mixed effects model). Our findings suggest sSNVs in the brain may constitute an additional component of the complex genetic architecture of SCZ. This perspective argues for the need to further investigate somatic variation in the brain as an explanation of the discordance in monozygotic twins and a potential guide to the identification of novel therapeutic targets.
\end{abstract}

\section{Introduction}

Neural stem cells and neural progenitor cells produce tens of billions of neurons during the development of a healthy human brain ${ }^{1}$, with some estimates suggesting that a developing brain must produce, on average, $\sim 250,000$ new neurons every minute ${ }^{2}$. Although remarkably efficient, DNA replication and DNA repair are not

\footnotetext{
Correspondence: Panos Roussos (panagiotis.roussos@mssm.edu)

'Department of Psychiatry, Icahn School of Medicine at Mount Sinai, One Gustave L. Levy Place, New York, NY 10029, USA

${ }^{2}$ Department of Genetics and Genomic Sciences, Icahn School of Medicine at Mount Sinai, Institute for Genomics and Multiscale Biology, One Gustave L. Levy Place, New York, NY 10029, USA

Full list of author information is available at the end of the article.

These authors contributed equally: John F. Fullard, Alexander W. Charney
}

flawless processes; with a genome consisting of some $3 \times$ $10^{9}$ base pairs, errors in DNA replication and repair have been proposed to correspond to $\sim 1.3$ errors per cell division in the human brain ${ }^{3}$. In addition to replication and repair errors, non-inherited genetic variants can arise through a myriad of other molecular mechanisms ${ }^{4}$. As such, cells within an individual are genetically heterogenous and may contain an array of non-germline (or, "somatic") variants, including single-nucleotide variants (SNVs), indels ${ }^{5}$, as well as structural variants, such as copy number variants (CNVs), DNA breaks, inversions, and translocations ${ }^{6}$. Indeed, a study of single neurons from the human prefrontal cortex identified more than a thousand somatic SNVs (sSNVs) per cell ${ }^{7}$. If such mutations occur

\section{(c) The Author(s) 2019}

(c) (i) Open Access This article is licensed under a Creative Commons Attribution 4.0 International License, which permits use, sharing, adaptation, distribution and reproduction in any medium or format, as long as you give appropriate credit to the original author(s) and the source, provide a link to the Creative Commons license, and indicate if changes were made. The images or other third party material in this article are included in the article's Creative Commons license, unless indicated otherwise in a credit line to the material. If material is not included in the article's Creative Commons license and your intended use is not permitted by statutory regulation or exceeds the permitted use, you will need to obtain permission directly from the copyright holder. To view a copy of this license, visit http://creativecommons.org/licenses/by/4.0/. 
in critical genes, they may impact the function of the affected cells. Assuming it does not retard cell growth, the earlier a mutation arises during development, the more cells it will affect, and the more likely it is to lead to defects in tissue function and, ultimately, disease ${ }^{8}$.

The genetic architecture of schizophrenia (SCZ) is highly complex, with risk conferred through common variants ${ }^{9}$, de novo mutations ${ }^{10}$, rare $\mathrm{CNVs}^{11}$, and rare $\mathrm{SNVs}^{12,13}$. The concordance rate between monozygotic twins is in the range of $41 \%-65 \%^{14}$, suggesting noninherited factors also make significant contributions to disease risk. Somatic variation is one such potential factor; however, while extensive somatic variation in the brain is now a well-established phenomenon, its relevance to SCZ risk remains unclear. To that end, there is some recent evidence suggesting that somatic variation in the brain may play a role in $\mathrm{SCZ}$, including deletions ${ }^{15}, \mathrm{CNVs}^{16}$, and long interspersed element-1 (L1) retrotransposons ${ }^{17,18}$. Studies evaluating the contribution of sSNVs in the brain to the genetic architecture of SCZ are lacking.

Here, we report rates of sSNVs in the brain of SCZ cases and controls identified from high-coverage whole-exome sequencing (WES) of neurons and non-neurons isolated by fluorescence activated nuclear sorting (FANS) from frozen postmortem prefrontal cortex (PFC) specimens. Separation of neuronal and non-neuronal nuclei was facilitated by using an established neuronal specific antibody, anti-NeuN ${ }^{19}$, and a protocol that has been extensively used by our team ${ }^{20,21}$. We identified and validated a number of cell-type-specific somatic variants in PFC in $\mathrm{SCZ}$, and found a burden of SSNV in cases compared to controls that included higher rates of non-synonymous sSNVs. Genes affected by SCZ sSNVs were enriched for gene sets that have been implicated previously in SCZ by de novo SNVs and show prenatal-bias expression in human brain during neurodevelopment ${ }^{22}$. Although larger studies are necessary, our findings provide additional evidence that somatic mutations may contribute to SCZ and suggests new avenues of research toward better understanding and treatment of this common disorder.

\section{Materials and methods}

\section{Sample information}

Specimens were obtained from 20 subjects (nine cases, 11 controls) from the Mount Sinai NIH Brain Bank and Tissue Repository (NBTR) (Supplementary Table 1). All subjects were recently included in a large study of gene expression by the CommonMind Consortium ${ }^{23-25}$. In brief, the NBTR obtains brain specimens from the Pilgrim Psychiatric Center, collaborating nursing homes, Veteran Affairs Medical Centers, and the Suffolk County Medical Examiner's Office. Disease diagnoses are made based on DSM-IV criteria and are obtained through direct assessment of subjects using structured interviews and/or through psychological autopsy by extensive review of medical records and informant and caregiver interviews. Informed consent is obtained from the next of kin. The brain bank procedures are approved by the Icahn School of Medicine at Mount Sinai Institutional Review Board (IRB) and are exempt from further IRB review due to the collection and distribution of postmortem specimens.

\section{Tissue processing}

At autopsy, from each subject fresh frozen slabs were cut from the temporal muscle and Brodmann areas 9/46 of the left dorsolateral prefrontal cortex. Immediately after dissection, specimens were cooled to $-190^{\circ} \mathrm{C}$ and dry homogenized to a coarse powder using a liquidnitrogen-cooled mortar and pestle. The tissue was stored at $-80^{\circ} \mathrm{C}$ until processed.

\section{FANS of neuronal and non-neuronal nuclei}

Fifty milligrams of frozen brain tissue was homogenized in cold lysis buffer $\left(0.32 \mathrm{M}\right.$ sucrose, $5 \mathrm{mM} \mathrm{CaCl}_{2}, 3 \mathrm{mM}$ magnesium acetate, $0.1 \mathrm{mM}$, EDTA, $10 \mathrm{mM}$ Tris- $\mathrm{HCl}$, pH 8, $1 \mathrm{mM}$ DTT, $0.1 \%$ Triton X-100), and filtered through a $40-\mu \mathrm{m}$ cell strainer. The flow-through was underlaid with sucrose solution $(1.8 \mathrm{M}$ sucrose, $3 \mathrm{mM}$ magnesium acetate, $1 \mathrm{mM}$ DTT, $10 \mathrm{mM}$ Tris- $\mathrm{HCl}, \mathrm{pH} 8$ ) and subjected to ultracentrifugation at $24,000 \mathrm{rpm}$ for $1 \mathrm{~h}$ at $4{ }^{\circ} \mathrm{C}$. Pellets were re-suspended in $500 \mu \mathrm{l}$ DPBS supplemented with BSA (at a final concentration 0.1\%) and incubated with anti-NeuN antibody (1:1000, Alexa488 conjugated, Millipore cat. \#MAB377X) under rotation for $1 \mathrm{~h}$, at $4{ }^{\circ} \mathrm{C}$, in the dark. Prior to FANS sorting, DAPI (Thermoscientific) was added to a final concentration of $1 \mu \mathrm{g} / \mathrm{ml}$. DAPI positive NeuN+ (neuronal) and NeuN- (non-neuronal) nuclei were sorted into individual tubes, pre-coated with $5 \%$ BSA, using a FACSAria flow cytometer (BD Biosciences).

\section{DNA isolation and sequencing}

DNA was isolated from sorted nuclei using the Qiagen QIAamp DNA mini kit (cat. \#51306) according to manufacturer's instructions (Blood or Body fluid spin protocol). Similarly, DNA from temporal muscle samples was extracted using the Qiagen QIAamp DNA mini kit (DNA purification from tissues protocol). Purified DNA was quantified by Qubit (Life technologies) and submitted for WES sequencing (New York Genome Center). Samples were barcoded and pooled prior to enrichment for exonic DNA with the SureSelect Human All Exon V4 library. WES was performed on the HiSeq 2500 platform (Illumina, San Diego, CA, USA), producing 150 base-pair (bp) paired-end reads to a target depth of 250 reads per base for brain specimens and 50 reads per base for temporal muscle. 


\section{Sequence alignment and germline variant calling}

To facilitate alignment and germline SNV calling, we utilized a previously described in-house genome analysis pipeline composed from several widely used open source software projects ${ }^{26}$. In brief, short-reads were aligned to a build of the hg19 human reference genome masked for gender and pseudo-autosomal regions using bwa mem ${ }^{27}$. Indel realignment, de-duplication, and base-quality score recalibration (BQSR) were then implemented in accordance with "GATK Best Practices" guidelines. Germline SNVs were called with the GATK HaplotypeCaller ${ }^{28,29}$, and variant quality score recalibration (VQSR) was used to estimate the probability that a WES-identified germline SNV was a true variant instead of an artifact.

Per-individual quality control metrics were calculated from the output of alignment and germline SNV calling procedures. Low level contamination was assessed using VerifyBamID $^{30}$. Coverage metrics derived from the alignment data were calculated using Picard (http:// broadinstitute.github.io/picard) and germline SNV metrics derived from the Haplotypecaller output were calculated using PLINK/SEQ ${ }^{12}$. The latter included the total number of alternate alleles, mean heterozygosity, mean chromosome $\mathrm{X}$ heterozygosity, dbSNP percentages, and mean transition/transversion ratio at heterozygous sites. All samples were noted to have dbSNP percentages $>95 \%$, and all of the other metrics considered displayed broadly even profiles across samples (Supplementary Table 4) and thus were not used as the basis for further individual-level QC.

Identity concordance was performed between the three exomes labeled as being derived from the same individual. This was accomplished in PLINK ${ }^{31}$ using identity-by-state (IBS) and identity-by-descent (IBD) metrics derived from genotypes in a set of $\sim 5000$ SNPs in the WES data shown previously to be ancestry informative $\mathrm{e}^{12}$. These procedures led to the identification of a genetic mismatch between the temporal muscle and brain specimens of one individual, who was therefore excluded from the study. The input into sSNV calling algorithms therefore consisted of three exomes per individual (neurons from the brain, non-neurons from the brain, and temporal muscle) from 19 individuals (nine cases, 10 controls), for a total of 57 exomes.

\section{Somatic SNV calling and quality control}

We called sSNVs using MuTect ${ }^{32}$ (v1.1.6) and Strelka ${ }^{33}$ (v1.0.14) following a comparison of $6 \mathrm{sSNV}$ calling algorithms (Supplementary Information; Supplementary Figures 1-3). One non-neuronal brain specimen was removed due to an excess of sSNVs (Supplementary Information). Only sSNVs called by both algorithms were retained. This set was filtered using a conservative inhouse pipeline that kept only those putative sSNVs that met all of the following criteria: mapping quality $>10$, base quality $>10$, read depth $>10,2$ alleles observed, not a small insertion or deletion, $>10$ base pairs from another putative sSNV, $<350$ base pairs outside a target region in the exon capture kit, and minor allele frequency $<0.001$ (Supplementary Information). The quality and depth filters were required to be met in all three tissues for the individual with the putative sSNV. These initial quality-control procedures removed over $99 \%$ of the initial 18,522 sSNV calls made by MuTect and Strelka. The remaining 151 putative variants were then manually inspected with the Integrative Genomics Viewer (IGV; Supplementary Information $)^{34}$. Most ( 79\%) were determined to be likely artifact based on the manual inspection procedure, leading to a final set of $32 \mathrm{sSNVs}$ for downstream analyses (Supplementary Figure 4).

\section{Mutational signature analysis}

We performed signature analysis by estimating the frequency of mutations in their context for a trinucleotide substitution matrix using sSNVs from the current study (which we call "Fullard" signatures) and two studies conducted in single neurons by Lodato et al. ${ }^{35}$ ("Lodato" signatures) and Bae et $\mathrm{al}^{3}$ ("Bae" signatures). Mutation signatures were detected using the signeR package, which applies a Bayesian nonnegative matrix factorization-based mutational signature framework ${ }^{36}$. We run separate analysis in each study (Fullard, Lodato, and Bae) using the default signeR parameters. The number of signatures for each dataset was determined based on the maximization of the median Bayesian Information Criterion as implemented in the signeR package. We identified 1, 2, and 3 signatures for Fullard, Bae, and Lodato datasets, respectively. The identified signatures were clustered with the 30 COSMIC signatures (http://cancer.sanger.ac.uk/ cosmic/signatures), using unsupervised hierarchical clustering with correlation as the distance metric.

\section{Gene set enrichment analysis}

To define gene sets enriched for sSNVs in cases with SCZ compared to controls, we used Mutation Enrichment Gene set Analysis of Variants (MEGA-V; https://github. $\mathrm{com} /$ ciccalab/MEGA) $^{37}$. The SCZ and control gene sets were defined based on genes affected by sSNVs. We ran enrichment analysis using two different groups of gene sets:

\section{Hypothesis-free}

We performed exploratory analyses of a large number of gene sets derived from MsigDB 5.1 ${ }^{38}$, including: (i) Gene Ontology (GO) sets of molecular functions (MF), biological processes (BP), and cellular components (CC) (http://www.geneontology.org) ${ }^{39}$; (ii) Reactome database of pathways and reactions in human biology (http://www. 
reactome.org) ${ }^{40}$; (iii) Kyoto Encyclopedia of Genes and Genomes (KEGG) database (www.genome.jp/kegg) ${ }^{41}$; and (iv) Pathway Interaction Database (PID $)^{42}$. To enhance power, we limited the analysis to gene sets with 100-1000 genes.

\section{Hypothesis-driven}

In addition, we generated a group of gene sets derived from previous SCZ genetic findings, including: common ${ }^{9}$, rare copy number ${ }^{11}$ and de novo ${ }^{10}$ variants, as well as gene sets associated with rare variants ${ }^{12}$ (fragile X mental retardation protein $^{43}$ and postsynaptic density ${ }^{44}$ ) and prenatal and postnatal signatures defined based on BrainSpan data (http://www.brainspan.org/).

For both analyses, we only considered genes that were captured by the exon kit. We compared the two distributions of mutation counts between SCZ and controls using the Wilcoxon-rank sum test. The resulting $P$-values were corrected for multiple testing based on the Benjamini and Hochberg method. We also performed bootstrap analysis by random sampling with replacement. We report significant genes sets at FDR $\leq 0.1$ and success rate in bootstrapping (\%) $>99 \%$. The Haldane-Anscombe correction was applied to calculate the odds ratio when one of the cells has zero value.

\section{Statistical analysis to compare mutational burden across SCZ cases and controls}

We applied linear mixed-effects regression models to test the mutational burden in neuronal and non-neuronal sSNVs among cases with SCZ and controls. Covariates of interest (disease status, cell type, and the interaction of cell type by disease status) and confounds (sex and ancestry) were modeled as fixed effects while donor was modeled as random effects. This statistical model allows for accurate estimates of the means, variances, and significances of each covariate of interest while accounting for the increased uncertainty due donor effects. We tested each covariate of interest for difference from zero, based on a $t$-test using the Satterthwaite approximation on the degrees of freedom. We fit the linear mixed-effects regression models using the function lmer from the lme4 $\mathrm{R}$ package (v1.1-17) and the lmerTest package (v3.0-1) to perform the Satterthwaite corrected $t$-tests.

\section{Validation experiments}

A number of SNVs identified by WES were selected for validation by Sanger sequencing of cloned PCR products corresponding to the mutated regions and/or by TaqManbased digital PCR (dPCR).

\section{Sanger sequencing}

PCR primers were designed to amplify the region flanking the nucleotide of interest (Life technologies)
(Supplementary Table 2). Following PCR, reactions were resolved on $2 \%$ agarose gels and bands of the predicted molecular weight were excised and subjected to gel purification (Qiagen Minelute Gel Extraction Kit-Qiagen cat. \#28604). Purified PCR products were sub-cloned in to the zero blunt topo cloning vector (Thermo Fisher Scientific cat. \# K280020) according to manufacturer's instructions. No fewer than 94 colonies from each transformation reaction were then subjected to Sanger sequencing (Genewiz) and the presence or absence of the relevant SNV was determined.

\section{dPCR}

We used TaqMan-based dPCR to validate some of the putative SNVs identified by WES (Supplementary Table 3). Custom TaqMan SNP Genotyping Assays (Life technologies) were performed using the QuantStudio 3D digital PCR 20 Chip Kit v2 (life technologies cat. \#A26316) and the QuantStudio 3D digital PCR system.

\section{Results \\ Increased burden of sSNVs in SCZ}

We assessed the presence of sSNVs in neuronal and non-neuronal nuclei isolated by FANS from prefrontal cortex of nine cases with SCZ and 11 controls. From each sample, we obtained WES for DNA extracted from three sources: (i) neuronal $(\mathrm{NeuN}+$ ) nuclei, (ii) non-neuronal (NeuN-) nuclei, and (iii) a peripheral, non-brain tissue (temporal muscle) (Fig. 1). After alignment, variant calling, and assessment of genetic concordance across tissues labeled as coming from the same donor, we removed one individual due to a biobank sample swap resulting in no matched muscle specimen. The final dataset comprised nine cases with SCZ and 10 controls. For all samples, WES data for neuronal, non-neuronal, and temporal muscle were utilized with the exception of one case with SCZ (individual S2), where the non-neuronal data were excluded due to an implausible excess of somatic variants (Supplemental Materials). The demographics of the final cohort included in this analysis is described in Supplementary Table 1. Cases and controls had similar technical sequencing metrics, including total coverage, proportion of deeply covered targets, and overall proportion of nonreference alleles (Supplementary Table 4).

To identify sSNVs, for each locus, we compare the fraction of reads harboring the alternate allele (the "variant allele fraction", or VAF) in two specimens from the same individual: one specimen suspected to contain an sSNV (the "somatic" sample; in this case, neurons or nonneurons) and one specimen not suspected to contain an sSNV (the "reference" sample). sSNVs were identified based on the consensus of two somatic variant callers, selected among other callers as described in the Supplementary Information. A total of $32 \mathrm{sSNVs}$ were in the 


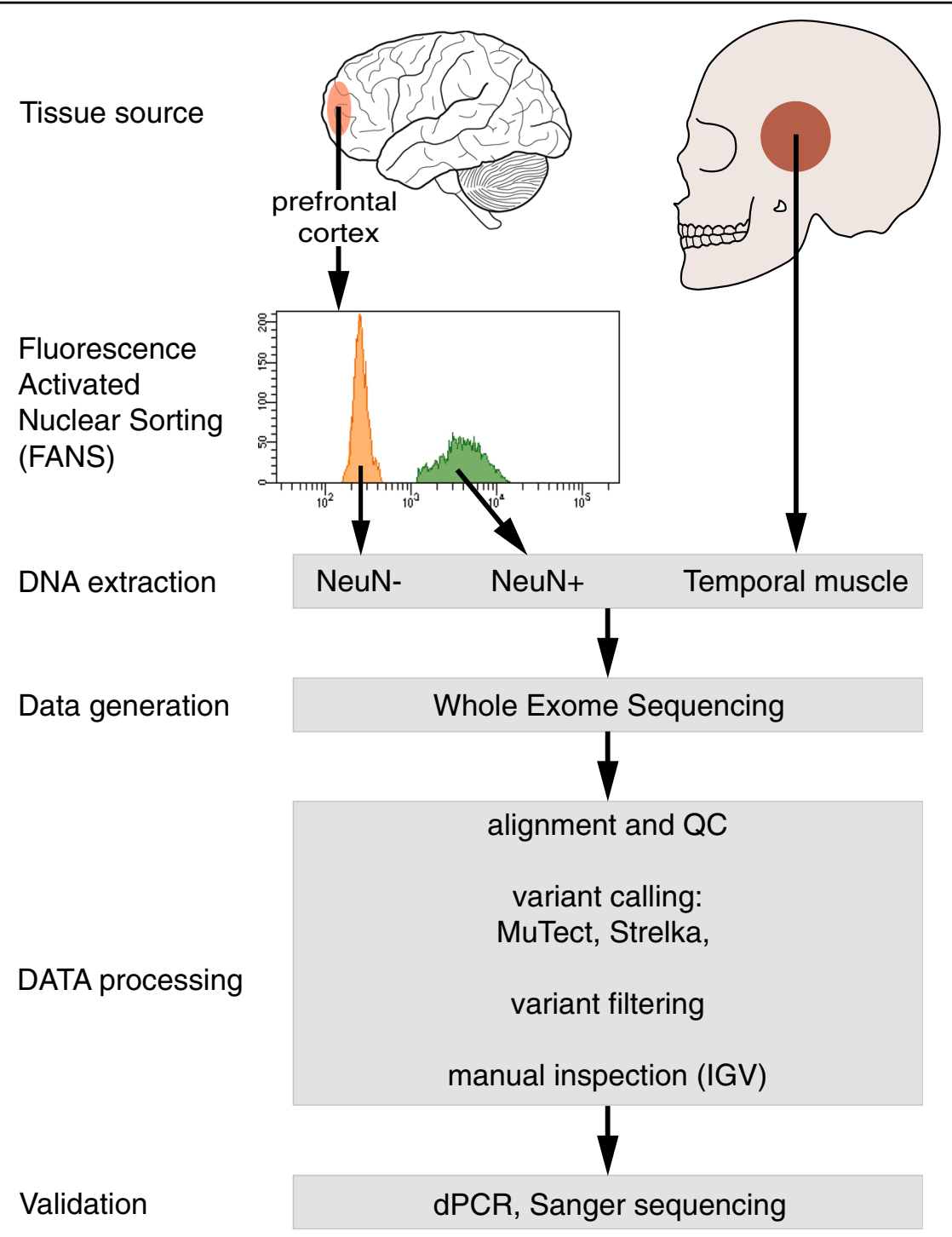

Fig. 1 Study design. Genomic DNA isolated from PFC derived neuronal (NeuN+) and non-neuronal (NeuN-) nuclei were subjected to wholeexome sequencing (WES), with DNA from temporal muscle used as an internal reference. Data were aligned to the genome and variants were identified using a combination of methods. Identified SSNVs were validated through direct sequencing of cloned PCR products corresponding to the genomic region of interest and/or by digital PCR (dPCR). QC quality control, IGV Integrative Genomics Viewer

final call-set, with 25 in cases (12 of which are predicted to be non-synonymous and loss-of-function) and seven in controls (five of which are predicted to be nonsynonymous and loss-of-function) (Table 1; Supplementary Figure 4). We detected a significant increase in sSNV burden in cases compared to controls $(P=0.0092$, linear mixed-effects model) (Fig. 2a and Supplementary Figure 5). No significant effect of cell type (neuron vs non-neuron) or interaction of cell type by case-control status was observed. The rate of non-synonymous (NS) and loss-offunction (LoF) sSNVs was 2.66-fold higher in SCZ compared to controls (SCZ rate $=1.33$, control rate $=0.5, P=$ 0.047, linear mixed-effects model; Table 1). All sSNVs were detected in a small proportion of cells. More specifically, on an average, $4 \%$ of the reads contained the somatic allele (range $2.2 \%-7.1 \%$ ), with no significant differences with regard to this metric in the sSNVs found among cases compared to those found among controls $(P=0.67, t$-test $)$ (Fig. $2 b)$.

\section{Mutational signature analysis}

Mutational signature analysis revealed a single signature explaining the mutational spectrum of SCZ and control sSNVs (Fig. 3a). This signature (which we call "Fullard") was comprised mainly of $\mathrm{C}>\mathrm{T}$ transitions. We found a larger fraction of $\mathrm{C}>\mathrm{T}$ transitions in SCZ resulting in a 


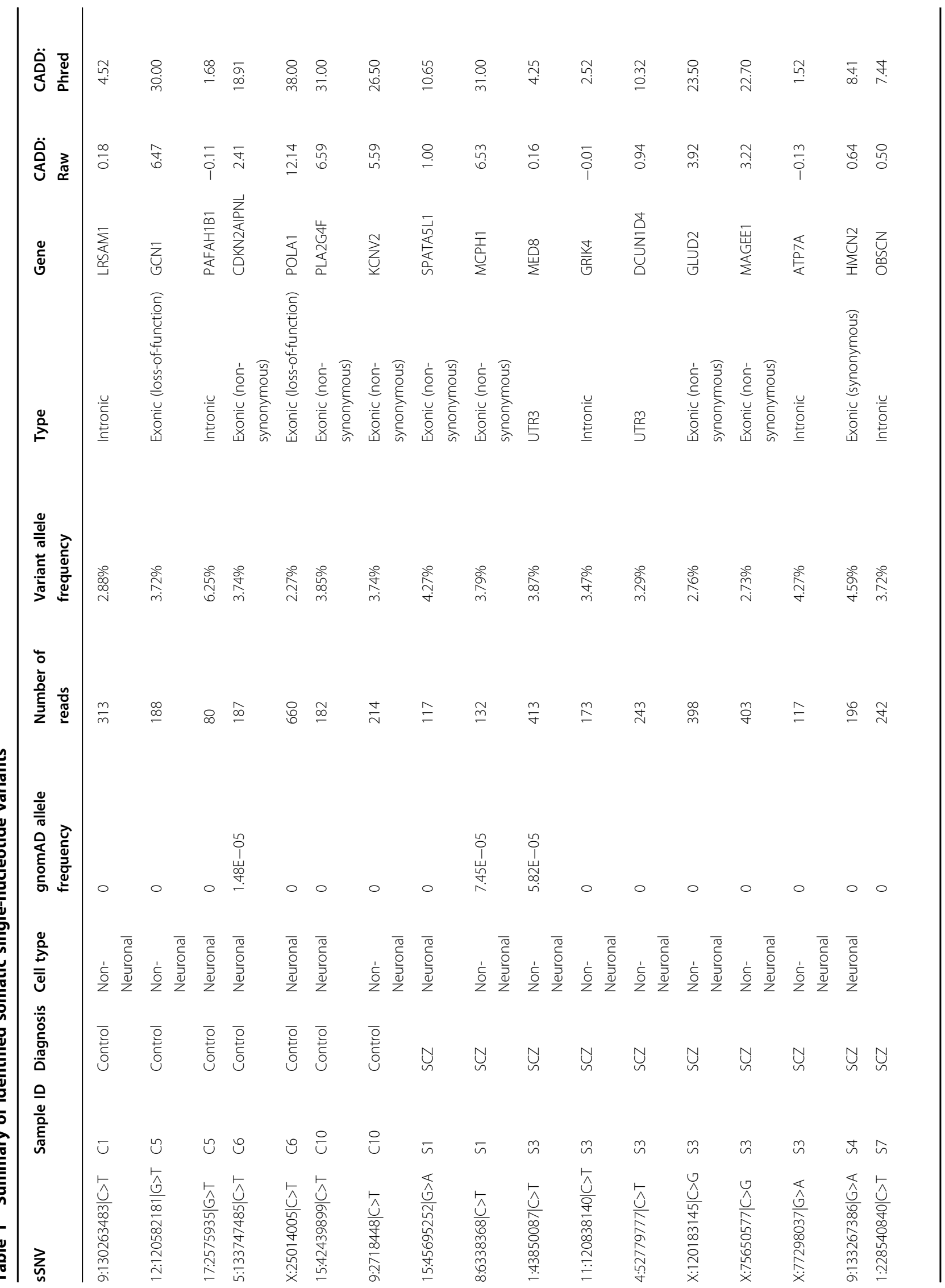




\begin{tabular}{|c|c|c|c|c|c|c|c|c|c|c|c|c|c|c|c|}
\hline 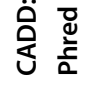 & $\stackrel{\grave{m}}{m}$ & $\underset{\stackrel{\circ}{0}}{\stackrel{2}{v}}$ & & $\underset{\infty}{\bar{\infty}}$ & $\underset{\infty}{\bar{\infty}}$ & $\begin{array}{l}\text { O } \\
\dot{m}\end{array}$ & $\begin{array}{l}\text { O } \\
\dot{m}\end{array}$ & $\begin{array}{l}\text { O } \\
\dot{m}\end{array}$ & $\bar{\forall}$ & $\underset{\Xi}{\equiv}$ & $\frac{\sigma}{\sigma}$ & $\stackrel{\stackrel{o}{\sim}}{\stackrel{i}{i}}$ & : & $\begin{array}{l}\stackrel{n}{+} \\
\stackrel{+}{+}\end{array}$ & ֻे \\
\hline 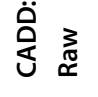 & $\stackrel{1}{\circ}$ & 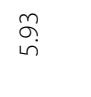 & $\stackrel{\stackrel{\leftrightarrow}{0}}{\circ}$ & ָิ & $\stackrel{\text { }}{\mathrm{i}}$ & $\stackrel{\grave{N}}{N}$ & 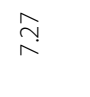 & $\stackrel{\circ}{\nwarrow}$ & $\frac{\overline{0}}{0}$ & $\stackrel{\infty}{\stackrel{\infty}{-}}$ & $\stackrel{\sim}{\tilde{c}}$ & 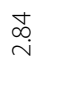 & $\underset{\sim}{8}$ & $\stackrel{+}{\stackrel{+}{*}}$ & $\underset{\mathrm{n}}{\mathrm{r}}$ \\
\hline ভٓ & 苍 & 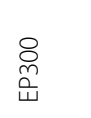 & & 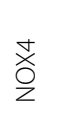 & 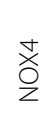 & $\stackrel{\infty}{\stackrel{\infty}{\xi}}$ & $\stackrel{\substack{0 \\
\sum}}{\sum}$ & 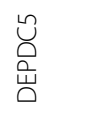 & 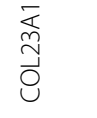 & $\begin{array}{l}\overline{\bar{\alpha}} \\
\bar{\infty} \\
\sum_{a}\end{array}$ & 岂 & $\frac{\sqrt{5}}{\frac{\pi}{4}}$ & 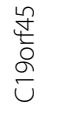 & $\stackrel{m}{\stackrel{m}{\breve{山}}}$ & $\begin{array}{l}\stackrel{n}{x} \\
\qquad\end{array}$ \\
\hline$\stackrel{0}{\gtrless}$ & $\begin{array}{l}\stackrel{\underline{\underline{E}}}{\underline{\underline{D}}} \\
\underline{\underline{E}}\end{array}$ & 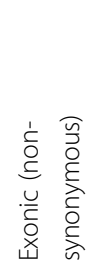 & 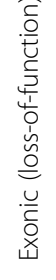 & 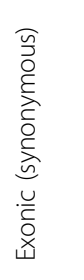 & 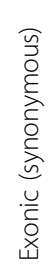 & 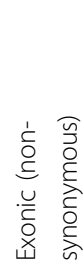 & 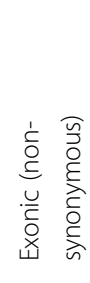 & 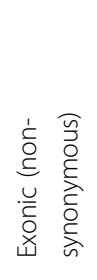 & $\begin{array}{l}\stackrel{U}{\underline{D}} \\
\stackrel{\underline{D}}{\underline{E}}\end{array}$ & 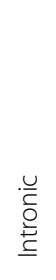 & 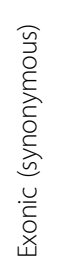 & 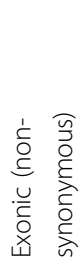 & 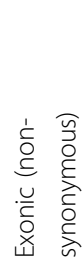 & 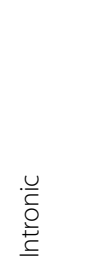 & 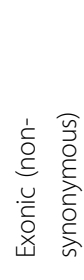 \\
\hline 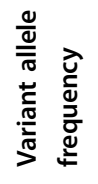 & 产 & $\begin{array}{l}\stackrel{\circ}{\circ} \\
\stackrel{+}{+}\end{array}$ & $\frac{\stackrel{\circ}{f}}{\frac{f}{+}}$ & $\frac{\stackrel{\circ}{\frac{0}{6}}}{\circ}$ & $\begin{array}{l}\text { ठे̀ } \\
\text { ơ }\end{array}$ & $\begin{array}{l}\text { ळे } \\
\stackrel{+}{+}\end{array}$ & ळे̀ & ষें & 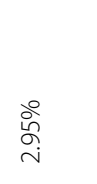 & 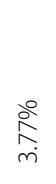 & $\underset{\text { \} }{\stackrel{\circ}{2}}} &{\text { 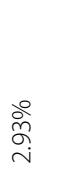 }} &{\text { 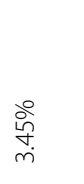 }} &{\begin{array}{l}\stackrel{\circ}{\circ} \\
\text { hn }\end{array}} &{\stackrel{\stackrel{\circ}{\stackrel{0}{\sim}}}{\stackrel{\sim}{\sim}}} \\
{\hline \text { 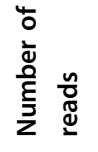 }} &{\stackrel{\infty}{\infty}} &{\stackrel{0}{ \pm}} &{\text { 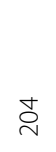 }} &{\stackrel{\infty}{\simeq}} &{\stackrel{\widetilde{\sigma}}{\circ}} &{\stackrel{m}{m}} &{\text { के }} &{\stackrel{n}{\sim}} &{\bar{\lesssim}} &{\stackrel{\text { n }}{\stackrel{n}{n}}} &{\stackrel{\widetilde{\sigma}}{ }} &{\stackrel{n}{\sim}} &{\stackrel{\text { 年 }}{\square}} &{\stackrel{\infty}{\circ}} &{\bar{q}} \\
{\hline \text { 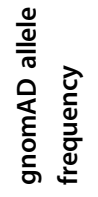 }} &{0} &{0} &{\text { 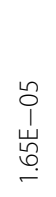 }} &{\text { 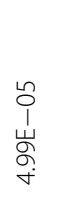 }} &{\text { 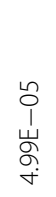 }} &{\text { 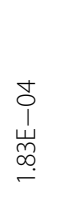 }} &{\text { 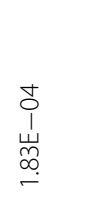 }} &{0} &{0} &{0} &{0} &{\begin{array}{l}\stackrel{u}{0} \\
1 \\
\stackrel{\leftrightarrow}{\aleph} \\
\end{array}} &{0} &{0} &{0} \\
{\hline \text { 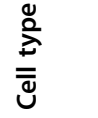 }} &{\text { 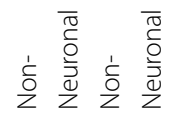 }} &{\text { 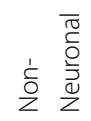 }} &{\begin{array}{l}\overline{0} \\
\overline{0} \\
\bar{O} \\
\bar{z}\end{array}} &{\text { 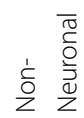 }} &{\begin{array}{l}\overline{0} \\
\overline{0} \\
\overline{\bar{O}} \\
\overline{0}\end{array}} &{\text { 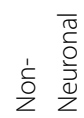 }} &{\text { 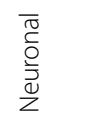 }} &{\text { 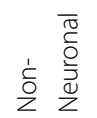 }} &{\text { 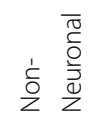 }} &{\begin{array}{l}\overline{0} \\
\overline{0} \\
\overline{0} \\
\bar{z}\end{array}} &{\text { 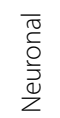 }} &{\text { 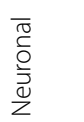 }} &{\text { 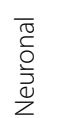 }} &{\text { 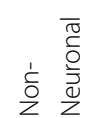 }} &{\text { 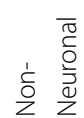 }} \\
{\hline \begin{array}{l}\text { g } \\
\stackrel{\sigma}{0}\end{array}} &{\underset{\sim}{N}} &{\underset{\sim}{N}} &{\underset{\sim}{ }} &{\underset{\sim}{N}} &{\underset{\sim}{N}} &{\underset{\sim}{N}} &{\underset{\sim}{\sim}} &{\underset{\sim}{N}} &{\underset{\sim}{N}} &{\underset{\sim}{N}} &{\underset{\sim}{ }} &{\underset{\sim}{~}} &{\underset{\sim}{\sim}} &{\underset{\sim}{N}} &{\underset{\sim}{N}} \\
{\hline n} &{\tilde{n}} &{\hat{n}} &{\hat{n}} &{\stackrel{\infty}{\sim}} &{\stackrel{\infty}{\sim}} &{\stackrel{\infty}{\sim}} &{\stackrel{\infty}{\sim}} &{\stackrel{\infty}{\sim}} &{\stackrel{\infty}{\sim}} &{\text { à }} &{\text { ڤs }} &{\text { }} &{\text { à }} &{\stackrel{a}{n}} &{\stackrel{n}{n}} \\
{\hline \sum_{\tilde{W}}} &{\text { 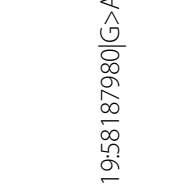 }} &{\text { 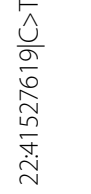 }} &{\text { 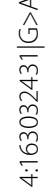 }} &{\text { 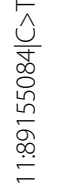 }} &{\text { 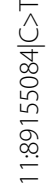 }} &{\text { 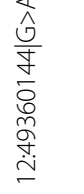 }} &{\text { 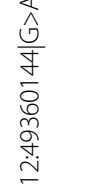 }} &{\begin{array}{l}\frac{N}{\hat{N}} \\
\stackrel{N}{N} \\
\infty \\
o \\
\stackrel{N}{N} \\
\stackrel{\sim}{N}\end{array}} &{\text { 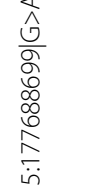 }} &{\text { 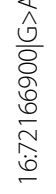 }} &{\text { 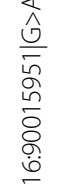 }} &{\text { 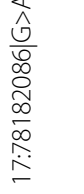 }} &{\text { 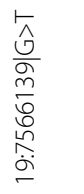 }} &{\text { 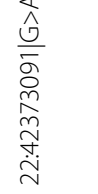 }} &{\text { 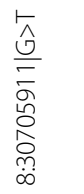 }} \\
$\hline
\end{tabular}


higher transition to transversion $(\mathrm{Ti} / \mathrm{Tv})$ ratio of 5.25 , compared to 2.5 in controls. We note that there was no case-control difference in $\mathrm{Ti} / \mathrm{Tv}$ ratio for germline variants called from the same data (average is 2.32 for both cases and controls; Supplementary Table 4). We then compared our results with mutational signatures derived from three studies: two sSNVs studies conducted in single neurons by Lodato et al. ${ }^{35}$ ("Lodato" signatures) and Bae et $\mathrm{al}^{3}$ ("Bae" signatures) and one study in cancer ${ }^{45}$ ("COSMIC" signatures). Unsupervised cluster analysis indicates a different mutational mechanism of "Fullard" with the other 2 brain signatures (Fig. 3b). The "Fullard" signature clusters with COSMIC 1,6 , and 15 signatures, which are related to endogenous mutational process initiated by spontaneous deamination of 5 -methylcytosine

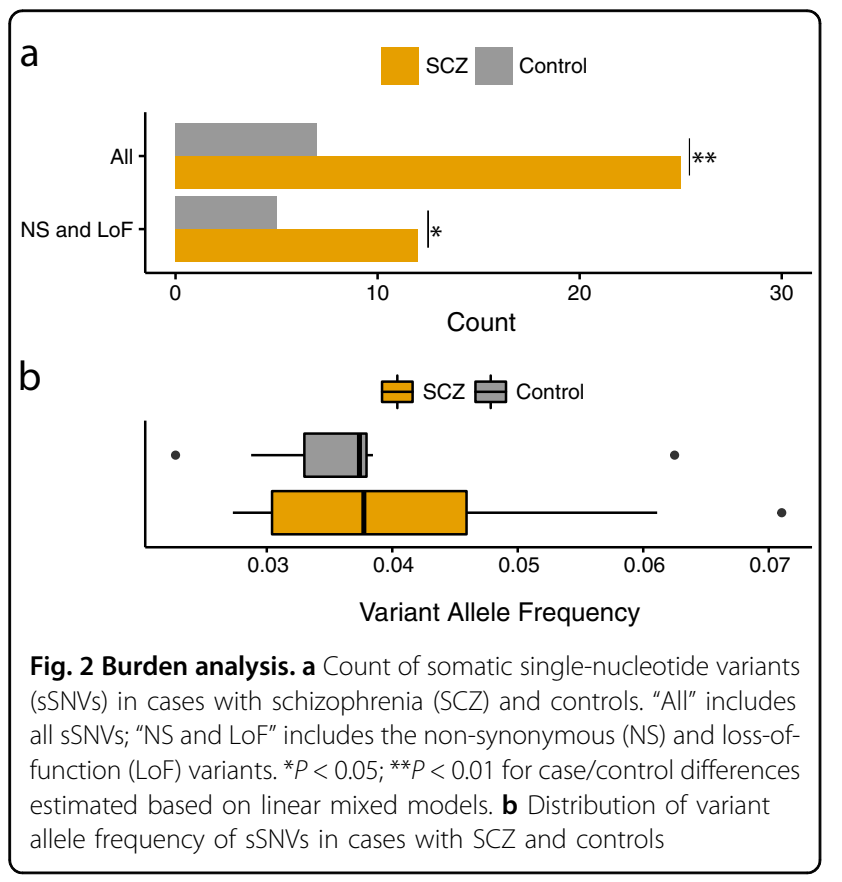

and defective DNA mismatch repair mechanisms. On the other hand, the "Bae 2" and "Lodato 2" cluster with COSMIC 5 and 16 signatures and resemble a "clock-like signature", as it was found in nearly all cancer COSMIC samples ${ }^{45}$. "Bae 1" and "Lodato 3" signatures comprise mostly of $\mathrm{C}>\mathrm{A}$ transversions and they are most closely associated with oxidative DNA damage. The different mutational signatures among our study and the previous studies $^{3,35}$ might be explained by the developmental stage at which the mutations arose. The sSNVs identified in our analysis were present, on average, in $4 \%$ of the cells in the specimen, indicating an early incident during development. The "Lodato" and "Bae" signatures derive from single cell sequencing and, therefore, will be detected independent of the developmental stage in which that mutation occurred, i.e. the threshold for detection of a somatic variant at the single cell level is lower than in our study.

\section{Gene set enrichment analysis}

We evaluated enrichment of genes affected by sSNVs in SCZ vs controls using two different groups of gene sets: (i) hypothesis driven, which includes genes previously implicated in SCZ, and (ii) hypothesis free, including gene sets related to biological pathways and molecular functions (see Methods for more details). In the hypothesisdriven set, we found enrichment for two gene sets (de novo mutations and prenatal genes) with SCZ that had odds ratio $>5$ and survived multiple testing corrections (Table 2). We observed enrichment SCZ sSNVs among the 854. SCZ de novo genes (four SCZ sSNVs in four SCZ de novo genes, zero control sSNVs in SCZ de novo genes; $P=0.026$, odds ratio $=8.6$ ). Similarly, the 1427 prenatalbiased genes were significantly enriched in SCZ sSNVs (three SCZ sSNVs in three pre-natal genes, zero control sSNVs in pre-natal genes; $P=0.026$, odds ratio $=6.7$ ). There was no significant enrichment of SCZ sSNVs with

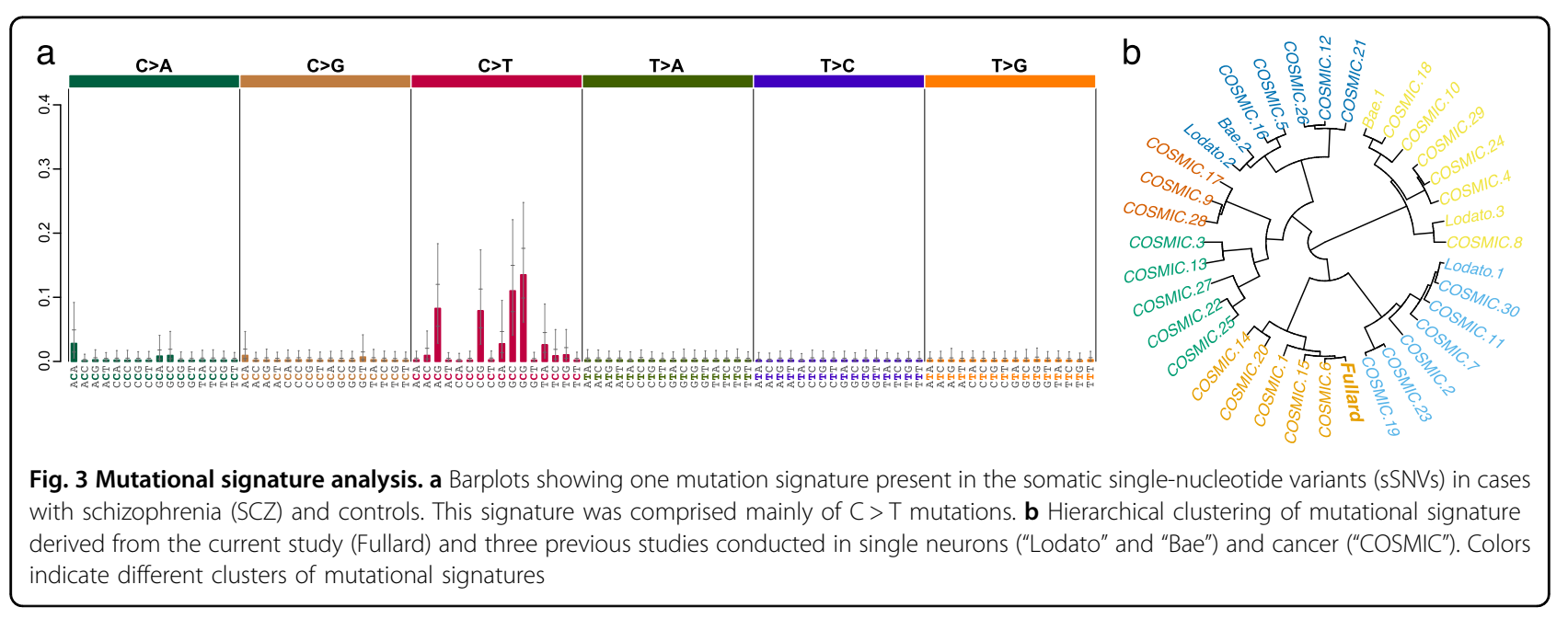




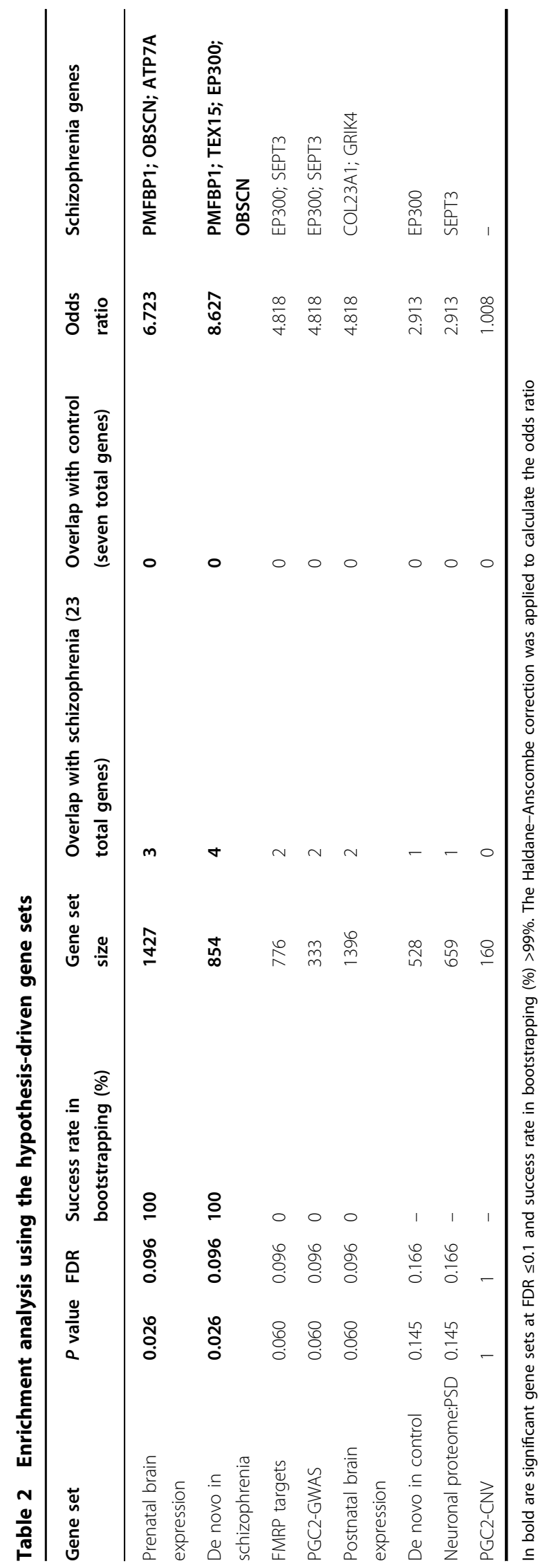

hypothesis free gene sets after multiple testing correction (Supplementary Table 5).

Validation of sSNVs in using dPCR and Sanger sequencing We selected seven NS sSNVs from cases with SCZ for further analysis based on availability of DNA from the same preparation used for WES analysis. Of these, four (in the genes encoding WNT1OB, DEPDC5, GLUD2, and $M A G E E 1)$ were successfully validated by quantitative dPCR (Fig. 4 and Supplementary Figure 6; Supplementary Table 3). To further validate our findings, we sought to confirm a selection of the identified sSNVs by Sanger sequencing of PCR products from multiple clones (cloneseq) designed to span the mutated nucleotide (Supplementary Table 2). We were particularly interested in confirming the mutation identified in DEPDC5, as its detection rate by dPCR was low $(0.4 \%)$ and may have been attributed to the background (Supplementary Figure 6). By clone-seq, the mutations affecting both DEPDC5 and $M A G E E 1$ were found in the genome of non-neuronal nuclei, at rate of $1.0 \%$ and $3.1 \%$, respectively. As with the dPCR experiment, the WNT1OB mutation was found in genomic DNA isolated from both neurons $(4.3 \%)$ and non-neurons (5.3\%) (Fig. 4b).

Next, we sought to examine the extent of the clones of mutant cells within the prefrontal cortex. Using independent, adjacent, dissections $(0.8 \mathrm{~cm}$ posterior to original dissection) from the same individuals, we isolated genomic DNA from neuronal and non-neuronal nuclei, as before. The presence of mutations identified in the original dissections was determined by $\mathrm{dPCR}$. Of the four mutations tested (sSNVs within WNT1OB, DEPDC5, GLUD2, and MAGEE1), only that affecting WNT10B was found in the secondary dissection (Fig. 4c and Supplementary Figure 7) As in the initial experiment, the WNT10B mutation was observed in the genome of both neurons and non-neurons, albeit at a lower rate $(10.4 \%$ and $4.5 \%$ vs $3.3 \%$ and $1.8 \%$, respectively) (Supplementary Table 6).

Overall, we were able to validate $57 \%$ (four out of seven) of sSNVs detected by our bioinformatic analysis using two independent experimental approaches. We failed to detect three of the four validated sSNVs in an additional, adjacent, dissection, indicating that those sSNVs are restricted to discrete clones of cells.

\section{Discussion}

Somatic variation is a major driver of disease, particularly in the context of cancer, and increasing evidence suggests a link between somatic mutation and neurological disorders ${ }^{5,6}$. Single cell approaches have begun to shed light on the contribution of somatic variation to the genetic heterogeneity of the human brain ${ }^{3,7,35,46-49}$. Each neuron in a healthy human brain is estimated to harbor 


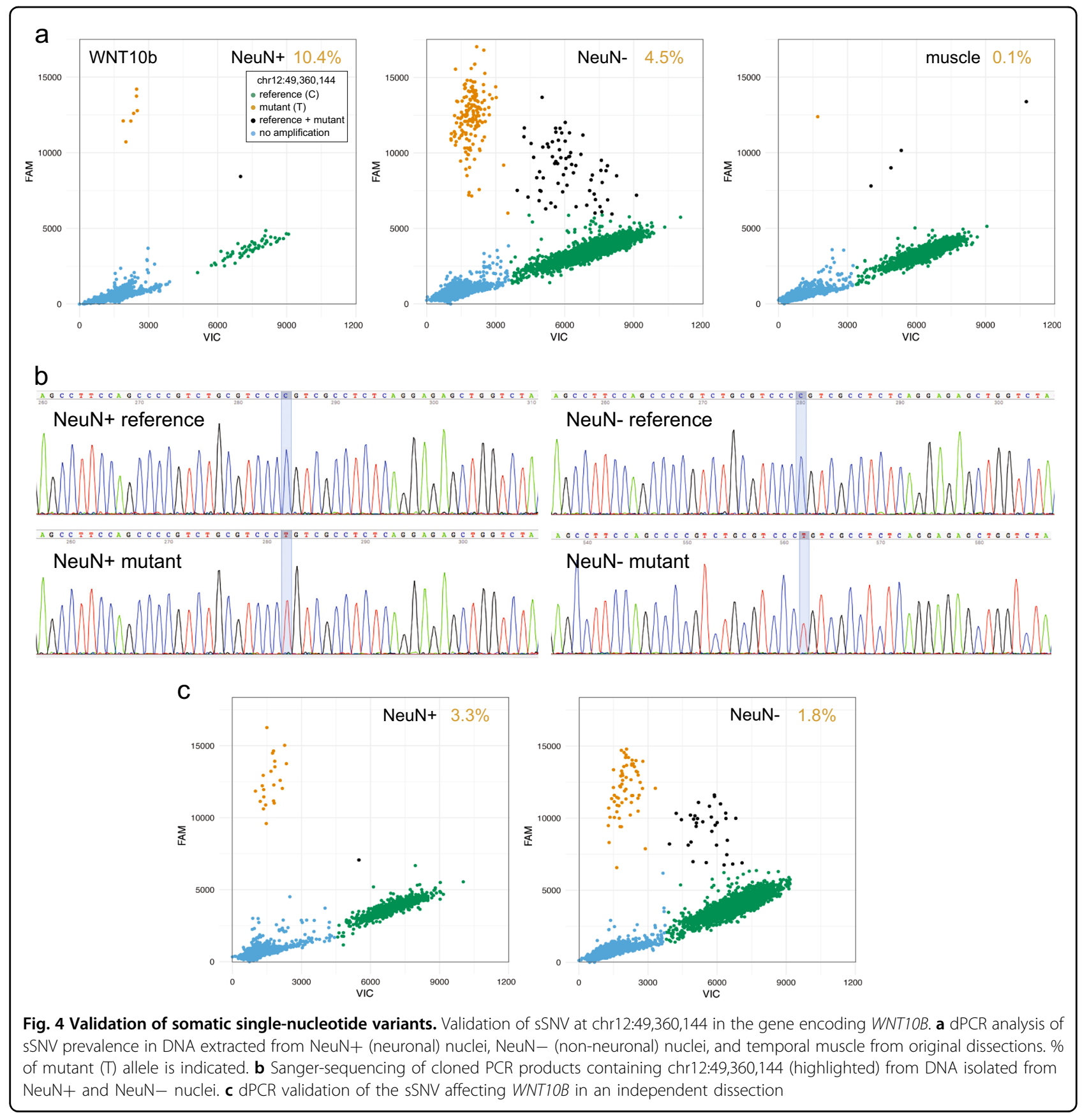

$>1000 \mathrm{SNVs}^{7}$. An increasing number of studies provide evidence in support of the notion that somatic variation contributes to defects in brain development and plays a role in neuropsychiatric disease (reviewed in ref. ${ }^{6}$ ).

The genetic etiology of SCZ is complex, but a number of recent large-scale studies have made significant progress toward understanding the contribution of common and rare variants to the disease ${ }^{9-13}$. Given the frequency at which monozygotic twins are discordant for $\mathrm{SCZ}^{50}$, it is plausible that not all SCZ risk variants are inherited through the germline. Previously, an increased burden of somatic deletions ${ }^{15}, \mathrm{CNVs}^{16}$, and long interspersed element-1 (L1) retrotransposons ${ }^{17,18}$ have been implicated in SCZ. Thus, we sought to assess the prevalence of sSNVs in postmortem brain specimens isolated from controls and individuals with SCZ. We reasoned that, for a sSNV to contribute to disease, it must meet a number of criteria: (1) the mutation must affect a critical gene but be non-cell lethal (i.e. the cell must be able to propagate despite the variant), (2) cells hosting the mutation must be relevant to the pathophysiology of the disease, and (3) those mutated cells must be sufficiently abundant at the 
time of death to affect a critical number of cells within the affected tissue. We compared the exome sequence of bulk DNA isolated from neurons, non-neurons, and a peripheral, non-brain tissue (temporal muscle). We identified 32 somatic variants ( 25 in cases, seven in controls). Of the mutations identified, seven were selected in order to validate our approach, of which four were confirmed.

One such variant was detected in both neurons and non-neurons of a SCZ case and affected the gene encoding $W N T 10 B$. Wnt signaling plays a critical role in a broad array of cellular process including cell fate determination, polarity, and cell adhesion ${ }^{51}$. With respect to brain function, Wnt signaling has been found to mediate axon guidance, fasciculation, and neural development ${ }^{52,53}$. Numerous studies link aberrant Wnt signaling and $\mathrm{SCZ}^{54-56}$. We also identified a SCZ mutation affecting non-neuronal cells in the gene encoding DEPDC5, which is an inhibitory component of the TORC1 pathway ${ }^{57}$. An increasing number of studies associate defective DEPDC5 function with epilepsy ${ }^{58}$ and a somatic mutation has been associated with focal cortical dysplasia ${ }^{59}$. An additional SCZ non-neuron SSNV was identified in the gene encoding the glutamate dehydrogenase, GLUD2. GLUD2 plays a number of important roles during neurotransmission, where it is involved in maintaining synapse integrity $^{60}$, the recycling of glutamate during neurotransmission $^{61}$ and has recently been shown to regulate burst firing of dopaminergic neurons ${ }^{62}$. Although the mutation we detected was restricted to non-neuronal cells, GLUD2 expression has been shown to be relatively high in astrocytes compared to neurons in the cerebral cortex, and has been hypothesized to facilitate the supportive role played by these cells in neuronal function ${ }^{63}$. Furthermore, activity of GLUD2 has previously been shown to be elevated in the prefrontal cortex of individuals with $\mathrm{SCZ}{ }^{64}$.

Of the $>1000$ SNVs to be found in each neuron of the human brain, the vast majority $(\sim 80 \%)$ have been shown to consist of $\mathrm{C}>\mathrm{T}$ transitions ${ }^{7}$. In the mouse, this number is $~ 100$ SNVs per neuron, of which $\sim 40 \%$ are $\mathrm{C}>\mathrm{T}$ transitions ${ }^{65}$. $\mathrm{C}>\mathrm{T}$ transitions have been associated with mutations that arise early in the developing brain ${ }^{3}$. Given that the sSNV affecting WNT1OB identified in this study was a $C>T$ transition, was found in both neurons and non-neurons, and was detected in two different, adjacent, tissue dissections, we conclude that this mutation occurred relatively early in development and, as such, is more likely to contribute to disease. In addition, these mutational signatures characteristic of early development are supported by our gene set enrichment analysis indicating enrichment with genes active in the prenatal brain (Table 2).

Our validation rate $(57 \%)$ is notably lower than those reported in cancer studies ${ }^{32}$. The allelic fractions in cancer biopsies, however, are typically higher ( 20\%) than those seen in our data. Even at the deep coverage used in this study (250x in the brain tissue), calling somatic variants when the fraction of cells harboring the variant is low (e.g. 5\%) relies on detection of a small number of reads harboring the variant allele. Future study designs might mitigate this issue through deeper sequencing and, as more data become available, through the utilization of computational approaches to assist in the identification of false positives ${ }^{66}$.

In this study, we chose to focus on exomes, as the potential impact of variants found therein is more readily discernible. Future studies should explore the somatic variation by applying whole-genome sequencing approaches, and include exploration of other variants such as L1 retrotransposons and structural variation. As our study included a relatively small number of samples, we are insufficiently powered to conclude that the greater number of non-synonymous somatic variants found in SCZ brain tissue is a characteristic of the disease. In addition, validation experiments using animal models are required to determine the phenotypic effects of identified mutations. One approach might be to assess the effect of suppression (or overexpression) of homologous candidate genes in Zebra fish, similar to experiments used in a previous study to determine the impact of modulation of SCZ candidate gene expression on neuroanatomy ${ }^{23}$. An additional approach could employ transposon or CRISPRCas9-mediated mutagenesis of brain organoids to determine the structure/function effects of specific somatic mutations ${ }^{67}$.

Despite the limitations of our study, we provide a methodology toward assessing the role played by somatic mutation in SCZ. Additional studies in twins discordant for SCZ, or including larger number of samples (such as those proposed by The Brain Somatic Mosaicism Network $^{6}$ ), may lead to the identification of previously unknown disease-associated genes or pathways and, in turn, to the discovery of novel therapeutic targets.

\footnotetext{
Acknowledgements

This work was supported by the National Institutes of Health—R01AG050986 (P.R.), R01MH109677 (P.R.), Brain Behavior Research Foundation (20540, P.R.), and the Veterans Affairs (Merit grant BX002395, P.R.). Further, this work was supported in part through the computational resources and staff expertise provided by Scientific Computing at the Icahn School of Medicine at Mount Sinai. The funders had no role in the design and conduct of the study; collection, management, analysis, and interpretation of the data; preparation, review, or approval of the manuscript; and decision to submit the manuscript for publication.
}

\section{Author details}

'Department of Psychiatry, Icahn School of Medicine at Mount Sinai, One Gustave L. Levy Place, New York, NY 10029, USA. ${ }^{2}$ Department of Genetics and Genomic Sciences, Icahn School of Medicine at Mount Sinai, Institute for Genomics and Multiscale Biology, One Gustave L. Levy Place, New York, NY 10029, USA. ${ }^{3}$ Sema4, 333 Ludlow Street, Stamford, CT 06902, USA. ${ }^{4}$ Mental 
IIIness Research, Education, and Clinical Center (VISN 2 South), James J. Peters VA Medical Center, Bronx, NY 10468, USA

\section{Conflict of interest}

The authors declare that they have no conflict of interest.

\section{Publisher's note}

Springer Nature remains neutral with regard to jurisdictional claims in published maps and institutional affiliations.

Supplementary information accompanies this paper at (https://doi.org/ 10.1038/s41398-018-0342-0).

Received: 2 August 2018 Revised: 15 September 2018 Accepted: 13 November 2018

Published online: 17 January 2019

\section{References}

1. Lui, J. H., Hansen, D. V. \& Kriegstein, A. R. Development and evolution of the human neocortex. Cell 146, 18-36 (2011).

2. Ackerman, S. Discovering the Brain (National Academy Press, Washington, DC, 1992).

3. Bae, T. et al. Different mutational rates and mechanisms in human cells at pregastrulation and neurogenesis. Science 359, 550-555 (2017).

4. Li, X. -Q. Molecular Mechanisms of Somatic Genome Variation. Somatic Genome Variation in Animals, Plants, and Microorganisms. pp. 337-350 (WileyBlackwell, 2017)

5. Lupski, J. R. Genetics. Genome mosaicism--one human, multiple genomes. Science 341, 358-359 (2013).

6. McConnell, M. J. et al. Intersection of diverse neuronal genomes and neuropsychiatric disease: The Brain Somatic Mosaicism Network. Science 356, 6336 (2017).

7. Lodato, M. A. et al. Somatic mutation in single human neurons tracks developmental and transcriptional history. Science 350, 94-98 (2015).

8. Campbell, I. M., Shaw, C. A., Stankiewicz, P. \& Lupski, J. R. Somatic mosaicism: implications for disease and transmission genetics. Trends Genet. 31, 382-392 (2015).

9. PGC-SCZ. Biological insights from 108 schizophrenia-associated genetic loci. Nature 511, 421-427 (2014).

10. Fromer, M. et al. De novo mutations in schizophrenia implicate synaptic networks. Nature 506, 179-184 (2014).

11. CNV and Schizophrenia Working Groups of the Psychiatric Genomics C. Contribution of copy number variants to schizophrenia from a genome-wide study of 41,321 subjects. Nat. Genet. 49, 27-35 (2017).

12. Purcell, S. M. et al. A polygenic burden of rare disruptive mutations in schizophrenia. Nature 506, 185-190 (2014).

13. Genovese, G. et al. Increased burden of ultra-rare protein-altering variants among 4,877 individuals with schizophrenia. Nat. Neurosci. 19, 1433-1441 (2016).

14. Cardno, A. G. \& Gottesman, I. I. Twin studies of schizophrenia: From bow-andarrow concordances to Star Wars Mx and functional genomics. Am. J. Med. Genet. 97, 12-17 (2000).

15. Kim, J. et al. Somatic deletions implicated in functional diversity of brain cells of individuals with schizophrenia and unaffected controls. Sci. Rep. 4, 3807 (2014).

16. Sakai, M. et al. Assessment of copy number variations in the brain genome of schizophrenia patients. Mol. Cytogenet. 8, 46 (2015).

17. Doyle, G. A. et al. Analysis of LINE-1 elements in DNA from postmortem brains of individuals with schizophrenia. Neuropsychopharmacology 42, 2602 (2017).

18. Bundo, M. et al. Increased I1 retrotransposition in the neuronal genome in schizophrenia. Neuron 81, 306-313 (2014).

19. Jiang, Y., Matevossian, A., Huang, H. S., Straubhaar, J. \& Akbarian, S. Isolation of neuronal chromatin from brain tissue. BMC Neurosci. 9, 42 (2008).

20. Fullard, J. F. et al. Open chromatin profiling of human postmortem brain infers functional roles for non-coding schizophrenia loci. Hum. Mol. Genet. 26 1942-1951 (2017).
21. Fullard, J. F. et al. An atlas of chromatin accessibility in the adult human brain. Genome Res. 28, 1243-1252 (2018).

22. Kang, $H$. J. et al. Spatio-temporal transcriptome of the human brain. Nature 478, 483-489 (2011).

23. Fromer, M. et al. Gene expression elucidates functional impact of polygenic risk for schizophrenia. Nat. Neurosci. 19, 1442-1453 (2016).

24. Hauberg, M. E. et al. Large-scale identification of common trait and disease variants affecting gene expression. Am. J. Hum. Genet. 100, 885-894 (2017).

25. Hauberg, M. E. et al. Differential activity of transcribed enhancers in the prefrontal cortex of 537 cases with schizophrenia and controls. Mol. Psychiatry (2018).

26. Linderman, M. D. et al. Analytical validation of whole exome and whole genome sequencing for clinical applications. BMC Med. Genomics 7, 20 (2014).

27. Li, H. \& Durbin, R. Fast and accurate short read alignment with BurrowsWheeler transform. Bioinformatics 25, 1754-60 (2009).

28. DePristo, M. A. et al. A framework for variation discovery and genotyping using next-generation DNA sequencing data. Nat. Genet. 43, 491-8 (2011).

29. McKenna, A. et al. The Genome Analysis Toolkit: a MapReduce framework for analyzing next-generation DNA sequencing data. Genome Res. 20, 1297-1303 (2010).

30. Jun, G. et al. Detecting and estimating contamination of human DNA samples in sequencing and array-based genotype data. Am. J. Hum. Genet. 91, 839-848 (2012).

31. Purcell, S. et al. PLINK: a tool set for whole-genome association and population-based linkage analyses. Am. J. Hum. Genet. 81, 559-575 (2007).

32. Cibulskis, $\mathrm{K}$. et al. Sensitive detection of somatic point mutations in impure and heterogeneous cancer samples. Nat. Biotechnol. 31, 213-219 (2013).

33. Saunders, C. T. et al. Strelka: accurate somatic small-variant calling from sequenced tumor-normal sample pairs. Bioinformatics 28, 1811-1817 (2012).

34. Robinson, J. T. et al. Integrative genomics viewer. Nat. Biotechnol. 29, 24-26 (2011).

35. Lodato, M. A. et al. Aging and neurodegeneration are associated with increased mutations in single human neurons. Science 359, 555-559 (2017).

36. Rosales, R. A., Drummond, R. D., Valieris, R., Dias-Neto, E. \& da Silva, I. T. signeR: an empirical Bayesian approach to mutational signature discovery. Bioinformatics 33, 8-16 (2017).

37. Gambardella, G., Cereda, M., Benedetti, L. \& Ciccarelli, F. D. MEGA-V: detection of variant gene sets in patient cohorts. Bioinformatics 33, 1248-1249 (2017).

38. Subramanian, A. et al. Gene set enrichment analysis: a knowledge-based approach for interpreting genome-wide expression profiles. Proc. Natl Acad. Sci. USA 102, 15545-15550 (2005).

39. Ashburner, $M$. et al. Gene ontology: tool for the unification of biology. The Gene Ontology Consortium. Nat. Genet. 25, 25-29 (2000).

40. Croft, D. et al. The Reactome pathway knowledgebase. Nucleic Acids Res. 42 (Database issue), D472-D477 (2014).

41. Kanehisa, M. \& Goto, S. KEGG: kyoto encyclopedia of genes and genomes. Nucleic Acids Res. 28, 27-30 (2000).

42. Schaefer, C. F. et al. PID: the Pathway Interaction Database. Nucleic Acids Res. 37 (Database issue), D674-D679 (2009).

43. Darnell, J. C. et al. FMRP stalls ribosomal translocation on mRNAs linked to synaptic function and autism. Cell 146, 247-261 (2011).

44. Kirov, G. et al. De novo CNV analysis implicates specific abnormalities of postsynaptic signalling complexes in the pathogenesis of schizophrenia. Mol. Psychiatry 17, 142-153 (2012).

45. Alexandrov, L. B. et al. Clock-like mutational processes in human somatic cells. Nat. Genet. 47, 1402 (2015).

46. Cai, $X$. et al. Single-cell, genome-wide sequencing identifies clonal somatic copy-number variation in the human brain. Cell Rep. 10, 645 (2015).

47. Evrony Gilad, D. et al. Single-neuron sequencing analysis of L1 retrotransposition and somatic mutation in the human brain. Cell 151, 483-496 (2012).

48. McConnell, M. J. et al. Mosaic copy number variation in human neurons. Science 342, 632-637 (2013).

49. Evrony, G. D., Lee, E., Park, P. J. \& Walsh C. A. Resolving rates of mutation in the brain using single-neuron genomics. elife 5, (2016).

50. Stabenau, J. R. \& Pollin, W. Heredity and environment in schizophrenia, revisited. The contribution of twin and high-risk studies. J. Ner. Ment. Dis. 181, 290-297 (1993).

51. Patapoutian, A. \& Reichardt, L. F. Roles of Wnt proteins in neural development and maintenance. Curr. Opin. Neurobiol. 10, 392-399 (2000). 
52. Nelson, W. J. \& Nusse, R. Convergence of Wnt, beta-catenin, and cadherin pathways. Science 303, 1483-1487 (2004).

53. Hur, E. M. et al. GSK3 controls axon growth via CLASP-mediated regulation of growth cone microtubules. Genes Dev. 25, 1968-1981 (2011).

54. Topol, A. et al. Altered WNT signaling in human induced pluripotent stem cell neural progenitor cells derived from four schizophrenia patients. Biol. Psychiatry 78, e29-e34 (2015).

55. Hennig, K. M. et al. WNT/beta-catenin pathway and epigenetic mechanisms regulate the Pitt-Hopkins Syndrome and schizophrenia risk gene TCF4. Mol. Neuropsychiatry 3, 53-71 (2017)

56. Hoseth, E. Z. et al. Exploring the Wnt signaling pathway in schizophrenia and bipolar disorder. Transl. Psychiatry 8, 55 (2018).

57. Hughes, J. et al. Knockout of the epilepsy gene Depdc5 in mice causes severe embryonic dysmorphology with hyperactivity of mTORC1 signalling. Sci. Rep. 7, 12618 (2017)

58. Baulac, S. Genetics advances in autosomal dominant focal epilepsies: focus on DEPDC5. Prog. Brain Res. 213, 123-139 (2014).

59. Ribierre, T. et al. Second-hit mosaic mutation in mTORC1 repressor DEPDC5 causes focal cortical dysplasia-associated epilepsy. J. Clin. Invest. 128, 2452-2458 (2018)

60. Elegheert, J. et al. Structural basis for integration of GluD receptors within synaptic organizer complexes. Science 353, 295-299 (2016).
61. Yamashita, M., Kawaguchi, S. Y. \& Hirano, T. Contribution of postsynaptic GluD2 to presynaptic R-type $\mathrm{Ca}(2+)$ channel function, glutamate release and longterm potentiation at parallel fiber to Purkinje cell synapses. Cerebellum $\mathbf{1 2}$, 657-666 (2013)

62. Benamer, N. et al. GluD1, linked to schizophrenia, controls the burst firing of dopamine neurons. Mol. Psychiatry 23, 691-700 (2018).

63. Spanaki, C., Zaganas, I., Kleopa, K. A. \& Plaitakis, A. Human GLUD2 glutamate dehydrogenase is expressed in neural and testicular supporting cells. J. Biol. Chem. 285, 16748-16756 (2010).

64. Burbaeva, G. S. et al. Glutamine synthetase and glutamate dehydrogenase in the prefrontal cortex of patients with schizophrenia. Prog. Neuropsychopharmacol. Biol. Psychiatry 27, 675-680 (2003).

65. Hazen, J. L. et al. The complete genome sequences, unique mutational spectra, and developmental potency of adult neurons revealed by cloning. Neuron 89, 1223-1236 (2016).

66. Kalatskaya, I. et al. ISOWN: accurate somatic mutation identification in the absence of normal tissue controls. Genome Med. 9, 59 (2017).

67. Bian, S. et al. Genetically engineered cerebral organoids model brain tumor formation. Nat. Methods 15, 631-639 (2018). 